\title{
SECOND NOTE ON FERMAT'S LAST THEOREM.
}

BY PROFESSOR R. D. CARMICHAEL.

IN a note printed on pages $233-236$ of the present volume of the Bulletin I have proved the following theorem:

If $p$ is an odd prime and the equation

$$
x^{p}+y^{p}+z^{p}=0
$$

has a solution in integers $x, y, z$ each of which is prime to $p$, then there exists a positive integer $s$, less than $\frac{1}{2}(p-1)$, such that

$$
(s+1)^{p^{2}} \equiv s^{p^{2}}+1 \bmod p^{3} .
$$

Professor Birkhoff has called my attention to the fact that condition (1) may be replaced by the simpler condition

$$
(s+1)^{p} \equiv s^{p}+1 \bmod p^{3},
$$

these two conditions being equivalent. Let us define the integers $\lambda$ and $\mu$ by the relations

Then

$$
(s+1)^{p}=s+1+\lambda p, \quad s^{p}=s+\mu p .
$$

(2)

We have also

$$
(s+1)^{p}=s^{p}+1+(\lambda-\mu) p .
$$

$$
\begin{aligned}
(s+1)^{p^{2}} & \equiv(s+1)^{p}+\lambda p^{2}(s+1)^{p-1} \bmod p^{3} \\
& \equiv s+1+\lambda p+\lambda p^{2} \bmod p^{3} \\
& \equiv s+1+\lambda\left(p+p^{2}\right) \bmod p^{3} .
\end{aligned}
$$

Likewise

$$
s^{p^{2}} \equiv s+\mu\left(p+p^{2}\right) \bmod p^{3} .
$$

From the last two congruences we have

$$
(s+1)^{p^{2}} \equiv s^{p^{2}}+1+(\lambda-\mu)\left(p+p^{2}\right) \bmod p^{3} .
$$

From (2) and (3) we see that a necessary and sufficient condition for either (1) or $\left(1^{\prime}\right)$ is that $\lambda-\mu \equiv 0 \bmod p^{2}$. Therefore (1) and $\left(1^{\prime}\right)$ are equivalent.

The simpler relation $\left(1^{\prime}\right)$ can be derived more readily than the relation (1). For from the congruence $x+y+z \equiv 0$ $\bmod p^{2}$, obtained in my previous paper, we have immediately $(x+y)^{p} \equiv-z^{p} \bmod p^{3}$. Hence 


$$
(x+y)^{p} \equiv x^{p}+y^{p} \bmod p^{3},
$$

from which $\left(1^{\prime}\right)$ is readily deduced.

Professor Birkhoff points out further that the test fails to be effective for all primes $p$ of the form $6 n+1$. For if $p=6 n+1$ it follows from the theory of primitive roots modulo $p^{3}$ that the congruence

$$
t^{3} \equiv 1 \bmod p^{3}
$$

has a solution $t$ for which $t-1$ is prime to $p$. Hence also

Then we have

$$
t^{2}+t+1 \equiv 0 \bmod p^{3} \text {. }
$$

$(t+1)^{p}=(t+1)(t+1)^{6 n} \equiv(t+1)\left(-t^{2}\right)^{6 n} \equiv t+1 \bmod p^{3}$, $(t+1)^{p^{2}} \equiv(t+1)^{p} \equiv t+1 \bmod p^{3}$,

and

$$
t^{p} \equiv t \cdot t^{6 n} \equiv t \bmod p^{3}, \quad p^{2} \equiv t^{p} \equiv t \bmod p^{3} .
$$

Therefore

Now put

$$
(t+1)^{p^{2}} \equiv t^{p^{2}}+1 \bmod p^{3} .
$$

Then

$$
t=\sigma+v p, \quad(0<\sigma<p-1) .
$$

$$
t^{p^{2}} \equiv \sigma^{p^{2}}, \quad(t+1)^{p^{2}} \equiv(\sigma+1)^{p^{2}} \bmod p^{3} .
$$

Therefore

$$
(\sigma+1)^{p^{2}} \equiv \sigma^{p^{2}}+1 \bmod p^{3}, \quad(0<\sigma<p-1) .
$$

This is relation (7) of my previous note; from this follows (1) as in the earlier treatment. Hence (1) is satisfied by all primes of the form $6 n+1$. Therefore the test can be useful only when the exponent $p$ is 3 or is of the form $6 n-1$.

INDIANA UNIVERSITY, March, 1913.

\section{AN EXTENSION OF A THEOREM OF PAINLEVÉ.}

BY DR. E. H. TAYLOR.

(Read before the American Mathematical Society, October 26, 1912.)

Theorem: Let $f(z)$ be a function which is single-valued and analytic throughout the interior of a region $S$ of the $z$-plane, $z=x+y i$. If $f(z)$ vanishes at every point of a 